\title{
Combined and selective miR-21 silencing and doxorubicin delivery in cancer cells using tailored DNA nanostructures
}

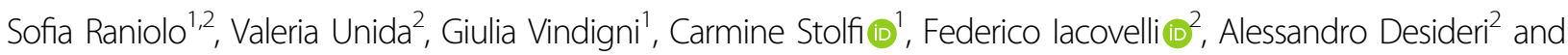 \\ Silvia Biocca (1D ${ }^{1}$
}

\begin{abstract}
MicroRNAs play an important role in tumorigenesis and, among them, miR-21 is found to be aberrantly up-regulated in various tumors. The tumor-associated antigen, folate receptor alpha is a GPI-membrane protein overexpressed in many malignant tumors of epithelial origin, including ovarian and cervical cancers. Covalently bound octahedral DNA nanocages were functionalized with folate molecules and utilized as scaffolds to engineer four sequestering units with a miR-21 complementary sequence for obtaining biocompatible Fol-miR21-NC non-toxic nanostructures, to be able to selectively recognize folate receptor alpha-overexpressing cancer cells and sequester the oncogenic miR-21. qPCR assays showed that Fol-miR21-NCs reduce the miR-21 expression up to $80 \%$ in cancer cells in the first 2 days of treatment. Functional assays demonstrated that miR-21 sequestering leads to up-regulation of miR-21 tumor suppressor targets (i.e., PTEN and Pdcd4), reduction in cancer cell migration, reduction in proliferation, and increase in cell death. Fol-miR21-NCs can be efficiently loaded with the chemotherapeutic agent doxorubicin. Co-delivery of antimiR-21 and doxorubicin showed additive cytotoxic effects on tumor cells, paving the way for their use as selective nucleic acid drugs.
\end{abstract}

\section{Introduction}

Aberrant expression of microRNAs (miRNAs) has been reported in various tumors indicating that there is a close correlation between miRNAs and human malignancy. MiRNAs are involved in many biological processes, such as cell proliferation and apoptosis, by regulating gene expression at post-transcriptional level ${ }^{1}$. Notably, dysregulation in the expression of different miRNAs contributes to cancer development and progression ${ }^{2-7}$. MiR21 was found to be consistently up-regulated in clinical samples from cancer patients ${ }^{8,9}$ and, in some cancer cell lines, it represents up to $15-25 \%$ of the total cellular

Correspondence: Silvia Biocca (biocca@med.uniroma2.it)

'Department of Systems Medicine, University of Rome Tor Vergata, Via Montpellier 1, 00133 Rome, Italy

2Department of Biology, University of Rome Tor Vergata, Via della Ricerca Scientifica 1, 00133 Rome, Italy

Edited by G. Melino
miRNA content ${ }^{10}$. Dysregulation of miR-21 expression is related with the proliferation, apoptosis, and migration of cancer cells ${ }^{9}$. The increase in miR-21 levels in cancer cells was found to down-regulate tumor suppressor proteins, PTEN and programmed cell death protein 4 (Pdcd4), and to regulate different apoptotic genes ${ }^{10}$. These observations suggest miR-21 knockdown as a promising anticancer therapeutic strategy. However, it is worth mentioning that miR-21 inhibition has been reported to be cytotoxic in several non-cancerous cell types ${ }^{11}$. Thus, to minimize off target side effects and avoid unexpected collateral damage in normal cells, a targeted therapy becomes crucial ${ }^{12}$.

The advent of nanotechnology in biomedicine has led to new opportunities in cancer therapy, offering the possibility to use innovative drug delivery systems ${ }^{13}$. DNAbased nanostructures (DNS) are at the forefront of emerging technologies for drug delivery, gene silencing,

\section{(c) The Author(s) 2021}

(c) Open Access This article is licensed under a Creative Commons Attribution 4.0 International License, which permits use, sharing, adaptation, distribution and reproduction cc) in any medium or format, as long as you give appropriate credit to the original author(s) and the source, provide a link to the Creative Commons license, and indicate if changes were made. The images or other third party material in this article are included in the article's Creative Commons license, unless indicated otherwise in a credit line to the material. If material is not included in the article's Creative Commons license and your intended use is not permitted by statutory regulation or exceeds the permitted use, you will need to obtain permission directly from the copyright holder. To view a copy of this license, visit http://creativecommons.org/licenses/by/4.0/. 
and diagnostic imaging as well as for studying molecular recognition and signal transduction processes ${ }^{14}$. Due to the intrinsic biocompatibility, structural flexibility, and stability of the polymer, DNA strands are good candidates for assembling DNS with different geometries and $\operatorname{sizes}^{15}$. DNS have been engineered to control switchable open/ close mechanisms and functionalized to include cellular recognition signals, such as folate, peptides, or aptamers, for generating nanostructures selectively targeting cancer cells through receptor-mediated mechanisms ${ }^{16-19}$. DNS have been also applied for miRNA detection, for overexpressed oncomiRs knockdown, or for tumor suppressive miRNA delivery ${ }^{20}$. Notably, neither intrinsic toxicity nor immune response have been detected after systemic injection of DNS into mouse models ${ }^{21}$. Our group has been deeply involved in the design, assembly, and structural-dynamical characterization of DNS with a specific geometrical arrangement, namely truncated octahedral DNA nanocages ${ }^{22,23}$, identifying the parameters that can modulate the yield of assembly and their mechanical-dynamical properties ${ }^{24}$. We characterized different types of fully covalently octahedral DNA nanocages and studied the receptor-mediated cell entry and their efficacy in selective drug delivery ${ }^{25-27}$. Among receptors, we focused on the $\alpha$-isoform of the folate receptor $(\alpha \mathrm{FR})$, a tumor-associated antigen highly expressed in many malignant tumors and largely absent in normal tissues. In general, folate-conjugation has great therapeutic potential for small RNAs and drug delivery, and for selective targeting of DNS to $\alpha$ FR-overexpressing cells ${ }^{28}$.

Doxorubicin (Dox) is one of the most efficacious chemotherapeutic agent used for a wide variety of solid tumors and hematological malignancies ${ }^{29}$. Dox intercalates into DNA double helix causing multiple toxic effects, such as prevention of DNA replication, disruption of topoisomerase-II-mediated DNA repair, and generation of reactive oxygen species ${ }^{30}$. Notably, DNS can be loaded with Dox and used as nanocarriers for drug delivery. In particular, folate-functionalized DNA nanocages (Fol-NCs) loaded with Dox induce a selective toxicity to cancer cells overexpressing the folate receptor ${ }^{26}$.

We recently obtained openable nanostructures that are able to recognize and bind specific oligonucleotide sequences in vitro and in cells, suitable for specifically sequestering intracellular oligonucleotides, such as miR$\mathrm{NAs}^{31}$. Here, we propose folate-functionalized NCs harboring DNA sequestering units complementary to miR21 (Fol-miR21-NCs), to form nanostructures with a specific miR-21 silencing activity. HeLa and IGROV1 cell lines were used as in vitro cervical and ovarian tumor models, since both cell lines overexpress miR-21 ${ }^{32,33}$ and present high levels of the $\alpha \mathrm{FR}^{26,34}$. We have analyzed time-dependent internalization, intracellular stability, targeting selectivity, and downstream gene regulation of Fol-miR21-NCs, evaluating their therapeutic efficacy following cell migration inhibition and cell death. Finally, Fol-miR21-NCs were loaded with Dox for evaluating the therapeutic efficacy of a combined treatment of miR-21 silencing and Dox release.

\section{Results}

\section{Models of the unbound/bound states of Fol-miR21-NC}

The Fol-miR21-NC was designed starting from a recently described H4-DNA cage ${ }^{31}$, where four miRNA sequestering units were introduced in a corner of a truncated octahedral DNA nanocage $\mathrm{e}^{22}$. The structure, resulting from the assembly of eight oligonucleotides, is characterized by a scaffold consisting of 12 doublestranded B-DNA helices, which form the main edges, connected by short single-stranded five-thymidine linkers, constituting the square truncated faces (Supplementary Fig. 1). The four sequestering units have 21-nucleotide DNA sequence, complementary to mature miR-21-5p, connected to the cage scaffold through two 12-nucleotide linkers made by random TC sequences. Binding of miRNAs induces a conformational change to the Fol-NC, switching it from an unbound (Fig. 1A, C) to a bound conformation (Fig. 1B, D). A 21-nucleotide random (scramble) DNA sequence was used to assemble a nonrelevant Fol-scr-NC to be used as negative control. For a selective receptor-mediated cell uptake, a single oligonucleotide has been functionalized with folate, to obtain folate-functionalized DNA nanocages ${ }^{26,27}$.

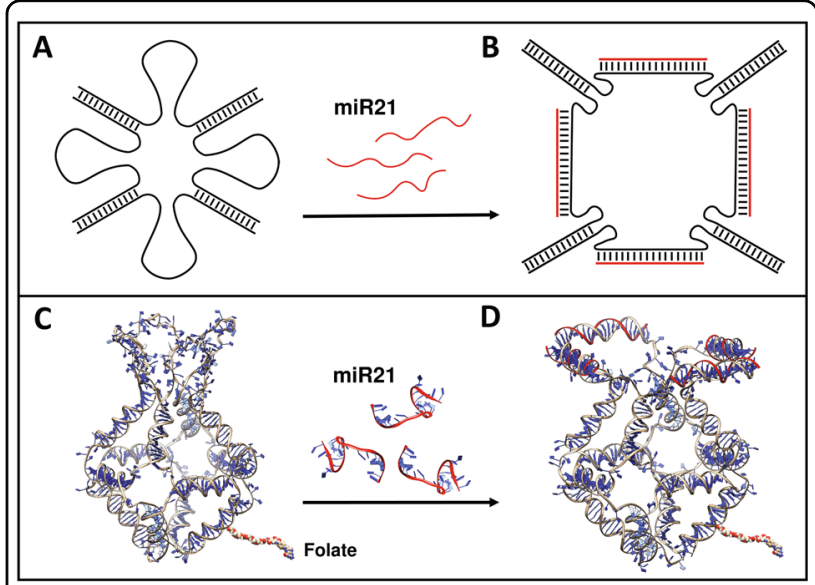

Fig. 1 Schematic and atomistic representation of Fol-miR21-NC. A Schematic top view of the four sequestering units in the unbound state. B Schematic top view highlighting the bound state of Fol-NC upon interaction with miR-21 molecules. C, D Three-dimensional representation of the two states, showing the conformational change occurring in the Fol-miR21-NC after binding to miR-21. 

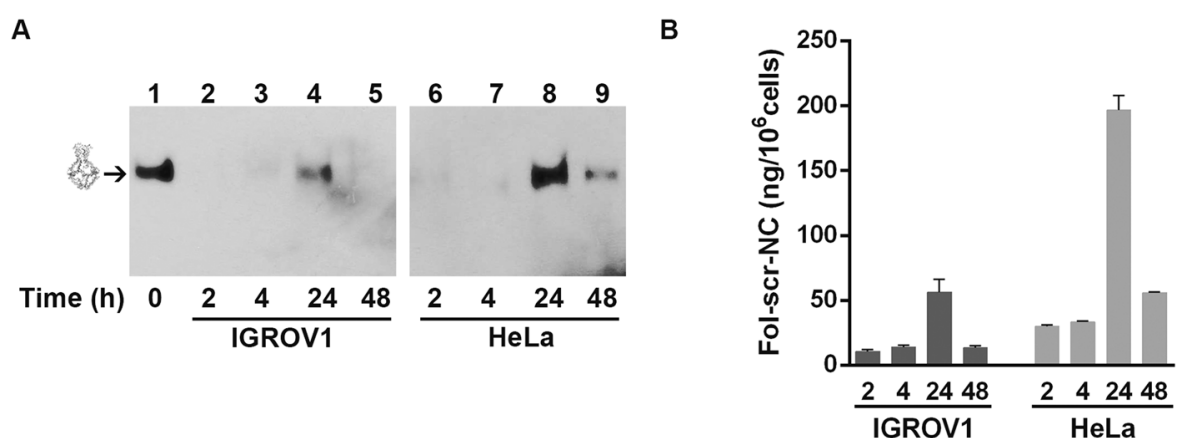

Fig. 2 Time-dependent uptake of Fol-scr-NCs in HeLa and IGROV1 cells. A DNA blot of biotinylated-Fol-scr-NCs purified from IGROV1 (lanes 2-5) and HeLa (lanes 6-9) cell lysates at 2, 4, 24, and $48 \mathrm{~h}$ incubation time and detected with streptavidin-HRP. Lane 1 shows $30 \mathrm{ng}$ of NCs before incubation with cells (time 0). B Densitometric analysis of the amount of Fol-scr-NCs internalized in cells at $37^{\circ} \mathrm{C}$ at different times, as indicated, analyzed with the ImageJ software. Histograms show average values \pm S.E.M. of three independent experiments.

\section{Internalization and stability of Fol-NCs in cancer cells}

Folate-functionalized nanostructures were assembled as described earlier ${ }^{22,25}$ and functionalized with a biotin molecule (Bio), for their detection through the biotinstreptavidin assays. To evaluate the time-dependent cellular uptake and stability of Fol-NCs, we used $\alpha$ FR-overexpressing HeLa and IGROV1 cells as in vitro tumor models ${ }^{26,34}$. It is worth mentioning that Fol-miR21-NCs and Fol-scr-NCs differ only for the four sequestering sequences. They are fully stable for at least $4 \mathrm{~h}$ in $10 \%$ FBS and then they slowly start to be degraded being still detectable at 24 h (Supplementary Fig. 2). As reported, the conformational change of Fol-NCs toward the open state leads to a lower degree of stability $^{31}$. Here we use Fol-scr-NCs as a model to initially study internalization and intracellular stability, since FolmiR21-NCs, once entered in the cells, undergo a conformational change toward the bound open state due to the intracellular presence of miR-21 (Fig. 1).

Fig. 2A shows the DNA blot of nanocages internalized via $\alpha$ FR pathway. In detail, HeLa and IGROV1 cells were incubated with $6 \mu \mathrm{g} / \mathrm{ml}$ of biotinylated Fol-scr-NCs for different times. It is important to remark that we did not observe any difference in the internalization mechanism using a range of concentrations between 1.5 and $10 \mu \mathrm{g} / \mathrm{ml}$ of nanostructures. Fol-NCs were purified from cell lysates and analyzed by DNA blot using streptavidin-HRP ${ }^{25}$. Lane 1 of Fig. 2A shows the electrophoretic mobility of the input band of nanocages $(30 \mathrm{ng}$ ) prior to the incubation with cells (time 0 ). A very low amount of Fol-scr-NCs is present after 2 and $4 \mathrm{~h}$ of incubation, while an evident band, corresponding to intact nanostructures, is detectable at $24 \mathrm{~h}$ in both IGROV1 and HeLa cells (Fig. 2A, lanes 4 and 8), confirming our previous observation that intact DNA nanocages accumulate inside the cells when uptaken by the folate-mediated pathway ${ }^{27}$. Of note, after $48 \mathrm{~h}$, intact Fol-scr-NCs are still detectable inside HeLa cells (lane 9), while they are almost undetectable in
IGROV1 cells (lane 5). The relative intensity of each DNA blot band was quantified by densitometric analysis, normalized to the intensity of the input and reported in Fig. 2B. The amount of internalized Fol-NCs after $24 \mathrm{~h}$ is $56.4 \pm 9 \mathrm{ng} / 10^{6}$ cells in IGROV1 and $196.5 \pm 11 \mathrm{ng} / 10^{6}$ cells in HeLa cells.

\section{Anti-miR-21 activity of Fol-miR21-NCs in folate receptor positive cancer cells}

To prove whether Fol-miR21-NCs, once entered in cells, sequester miR-21 thus decreasing its free concentration and inhibiting its activity, qPCR analysis of miR-21 expression was performed using the $\Delta \Delta \mathrm{Ct}$ method and normalized to the levels of endogenous U6 snRNA. The analysis in untreated cells shows a $23 \pm$ $6 \%$ higher expression level of miR-21 in HeLa compared to IGROV1 cells (Supplementary Fig. 3).

HeLa and IGROV1 cells were incubated with $1.5 \mu \mathrm{g} / \mathrm{ml}$ of Fol-NCs, repeating the treatment every day since, according to serum stability data (Supplementary Fig. 2), the lifetime of Fol-NCs is about $16-18 \mathrm{~h}^{31}$. After $24 \mathrm{~h}$ treatment with Fol-miR21-NCs, a selective inhibition of miR-21 expression was observed in both HeLa and IGROV1 cells $(15 \pm 3 \%$ and $34 \pm 9 \%$, respectively). A much higher inhibition was observed at $48 \mathrm{~h}$ and $72 \mathrm{~h}$, while, at longer time treatment ( 6 days), miR-21 level was not detectable. In detail, in HeLa cells the inhibition was $87 \pm 4 \%$ at $48 \mathrm{~h}$ and $92 \pm 3 \%$ at $72 \mathrm{~h}$ and in IGROV1 cells $70 \pm 8 \%$ and $80 \pm 6 \%$, respectively. Treatment of cells with non-relevant Fol-scr-NCs, used as negative control, for the same time intervals did not affect miR-21 intracellular level (Fig. 3).

To compare the Fol-miR21-NC's effects with an antimiR-21 oligonucleotide, we transfected HeLa cells with anti-miR-21 or a scramble oligonucleotide and observed a maximum of $66 \pm 6 \%$ decrease in miR-21 (Supplementary Fig. 5A). 

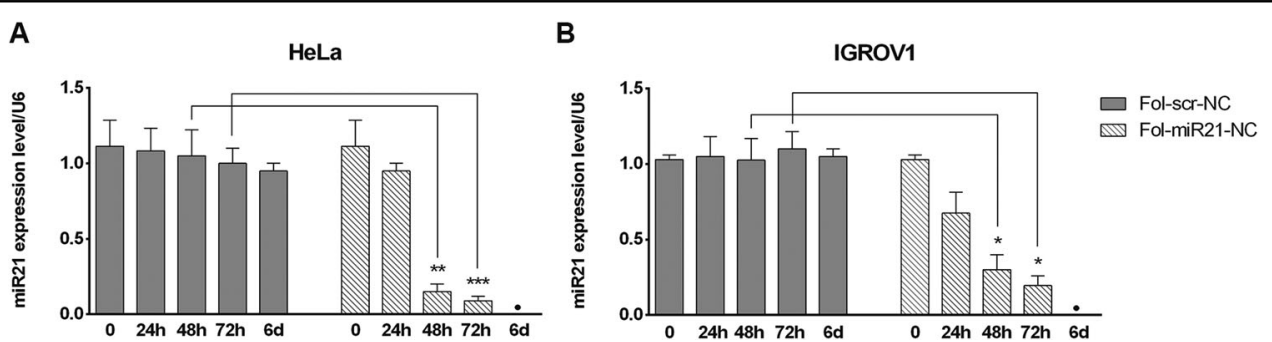

Fig. 3 MiR-21-silencing activity in HeLa and IGROV1 cells of Fol-miR21-NCs compared to Fol-scr-NCs. qPCR analysis of miR-21 expression level is reported for HeLa (A) and IGROV1 (B) cells incubated with Fol-NCs for different time intervals. Values are expressed as mean \pm S.E.M. of three different independent experiments. Statistical significance: ${ }^{*} P<0.05,{ }^{* *} P<0.01$, and ${ }^{* * *} P<0.001$ (Student's $t$-test). Values below detection limit (•).

A

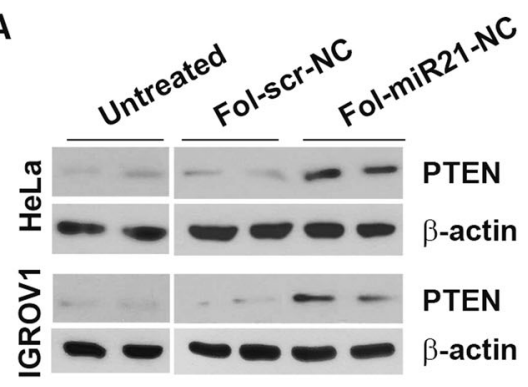

B

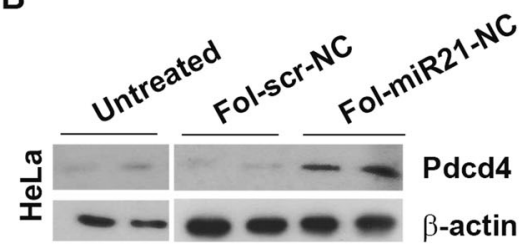

HeLa

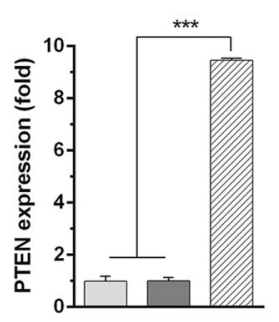

HeLa

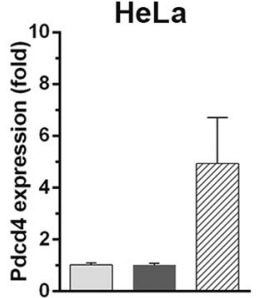

IGROV1

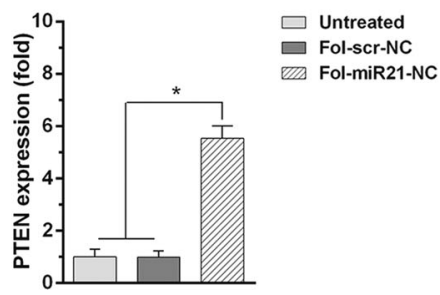

$\square$ Untreated

Fol-scr-NC

एय] Fol-miR21-NC

Fig. 4 Expression level of PTEN and Pdcd4 miR-21 target proteins. A Western blots of PTEN in HeLa and IGROV1 cells lysates, treated or not with Fol-NCs for $48 \mathrm{~h}$. B Western blot of Pdcd4 on lysates from HeLa cells treated or not with Fol-NCs for $72 \mathrm{~h}$. Densitometric analysis of three different experiments are shown on the right panels. Values are expressed as mean \pm S.E.M. Statistical significance: ${ }^{*} P<0.05$ and ${ }^{* * *} P<0.001$ (Student's $t$-test).

\section{Therapeutic effects of Fol-miR21-NCs on cancer cells}

To demonstrate the biological effects of miR-21 knockdown, we studied the expression level of PTEN and Pdcd4, two known downstream targets regulated by miR-21. HeLa and IGROV1 cells were incubated with $1.5 \mu \mathrm{g} / \mathrm{ml}$ Fol-miR21-NCs or Fol-scr-NCs, used as control, repeating the treatment every day. Figure 4A shows that the effect on PTEN expression by Fol-miR21-NCs is not evident after $24 \mathrm{~h}$ incubation, whereas a robust increase in PTEN level is observed after $48 \mathrm{~h}$. Densitometric analysis shows that PTEN level is $9.5 \pm 1$-fold higher in Fol-miR21-NC treated HeLa cells and $5.5 \pm 1$ fold higher in Fol-miR21-NC treated IGROV1 cells versus negative controls.

Up-regulation of Pdcd4 is detectable only after $72 \mathrm{~h}$ incubation in HeLa cells. Densitometric analysis indicates that Pdcd4 expression level is $4.9 \pm 2$-fold higher in
Fol-miR21-NC treated cells when compared to Fol-scr$\mathrm{NC}$ treated cells (Fig. 4B, right panel). No variation in the expression level of Pdcd4 protein was observed in IGROV1 cells. One reason could be that alterations in Pdcd4 protein expression are reported to be associated with the development of chemoresistance in ovarian cancer cell lines ${ }^{33}$. It is worth noting that untreated and Fol-scr-NC treated cells show comparable expression levels of PTEN and Pdcd4 proteins (Fig. 4 and Supplementary Fig. 4).

As a comparison, by transfecting cells with an anti-miR21 oligonucleotide for $24 \mathrm{~h}$, we observe a transient 2.5 -fold and 3-fold increase in PTEN level in HeLa and IGROV1 cells, respectively (Supplementary Figs. 5B and 6).

The effects of miR-21 knockdown on cell migration has been evaluated through a wound-healing assay on HeLa cells. Cells were treated with $1.5 \mu \mathrm{g} / \mathrm{ml}$ of Fol-NCs for 
A

$12 \mathrm{~h}$

$24 \mathrm{~h}$
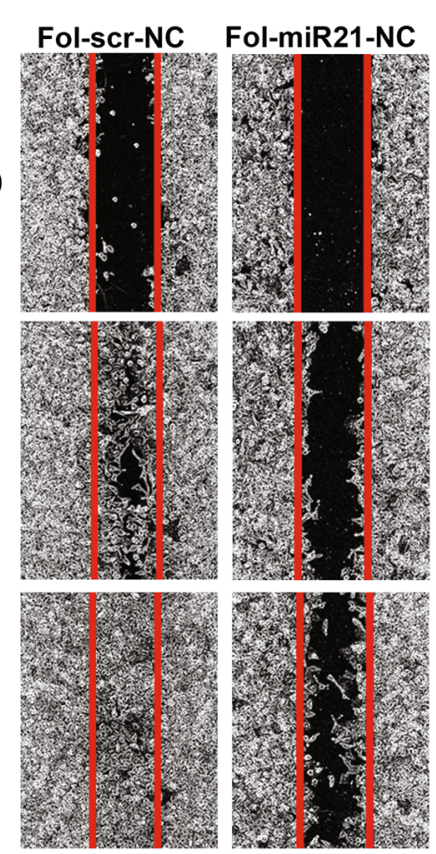

B

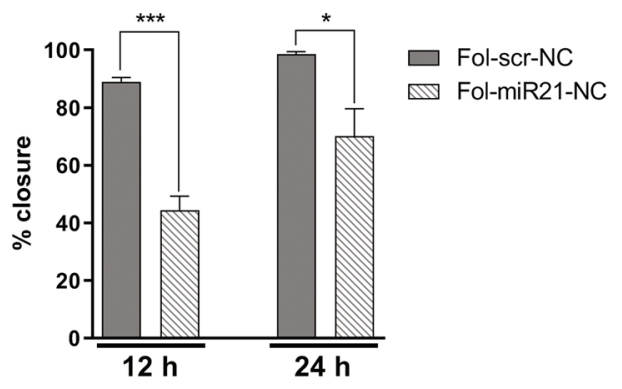

Fig. 5 MiR-21 silencing inhibits migration of HeLa cells. A In vitro wound-healing assay on cell migration across the scratched area was monitored for $24 \mathrm{~h}$. Images converted to grayscale are shown. B Histograms represent the relative percentage of distance covered by cells 12 and $24 \mathrm{~h}$ after scratch (\% closure). Values reported were obtained as mean \pm S.E.M. of three different experiments. Statistical significance: ${ }^{*} P<0.05$ and ${ }^{* * *} P$ $<0.001$ (Student's $t$-test).

$48 \mathrm{~h}$ and, after creating a wound, the scratch width was measured by photo recordings at 0,12 , and $24 \mathrm{~h}$. Fig. $5 \mathrm{~A}$ shows that Fol-miR21-NC treated cells were less efficient in migrating and closing the space into a confluent cell monolayer compared to scrambled control NCs. Quantitative histograms in Fig. $5 \mathrm{~B}$ show that, after $12 \mathrm{~h}$, the closure of the scratched area by Fol-miR21-NC treated cells is $44 \pm 5 \%$ compared to $89 \pm 2 \%$ of Fol-scr-NC treated cells and, after $24 \mathrm{~h}$, the closure is $70 \pm 9 \%$ and $98.5 \pm$ $1 \%$, respectively.

Anti-proliferative activity of Fol-miR21-DNA nanocages was tested by MTS assay, incubating HeLa and IGROV1 cells with $1.5 \mu \mathrm{g} / \mathrm{ml}$ nanocages for different times up to 8 days, repeating the treatment every day. Fig. $6 \mathrm{~A}$ shows that the reduction in cell proliferation after 3 days was $20 \pm 2 \%$ and $26 \pm 3 \%$, and reached $54 \pm 3 \%$ and $51 \pm 2 \%$ after 8 days of treatment in HeLa and IGROV1 cells, respectively. No effect was observed in cells treated with Fol-scr-NCs. In line with our previous observation ${ }^{26}$, Fol-miR21-DNA nanocages are selectively internalized in $\alpha \mathrm{FR}$-overexpressing cells and no reduction in cell proliferation was observed in cells that do not express the $\alpha F R s$ on their surface, such as human epidermoid carcinoma A431 or human embryonic kidney (HEK-293) cells (Supplementary Fig. 7). Notably, as expected, these cell lines do not internalize Fol-NCs (Supplementary Fig. 8).
On the other hand, in vitro cytotoxicity of Fol-miR21NCs determined by trypan blue assay shows an increase in cell death from the first day of incubation, up to $15 \%$ after 8 days (Fig. 6B). Notably, both HeLa and IGROV1 cells respond similarly to the treatment, notwithstanding the fact that IGROV1 cells internalize less than one-third of DNA nanocages compared to HeLa cells (Fig. 2).

\section{Combined treatment with Fol-miR21-NCs and doxorubicin}

We finally explored the role of Fol-miR21-NCs as multipurpose structures for miRNA silencing and as drug carriers by loading nanocages with Dox $^{26}$. By MTS assays we analyzed the cytotoxic effect of Fol-miR21-NCs, loaded or not loaded with Dox, in HeLa and IGROV1 cells (Fig. 7). Nanostructures were used at a basepair concentration of $4 \mu \mathrm{M}$. The estimated Dox concentration in Dox-loaded Fol-NCs was $0.44 \pm 0.1 \mu \mathrm{M}$, considering that $22.5 \%$ of the initial Dox used for intercalation is present in $\mathrm{NCs}$ after purification (Supplementary Fig. 9). At $72 \mathrm{~h}$ incubation, Fol-miR21-NC treatment led to a cell proliferation reduction of $15 \pm 4 \%$ and $22 \pm 3 \%$ over the control (Fol-scr-NCs) in HeLa and IGROV1 cells, respectively. Notably, treatment with Dox-loaded FolmiR21-NCs (Dox-Fol-miR21-NCs) induces an additive effect, which results in $45 \pm 4 \%$ reduction in cell viability in HeLa and $46 \pm 12 \%$ in IGROV1 cells. As expected, incubation of cells with control Fol-scr-NCs does not 

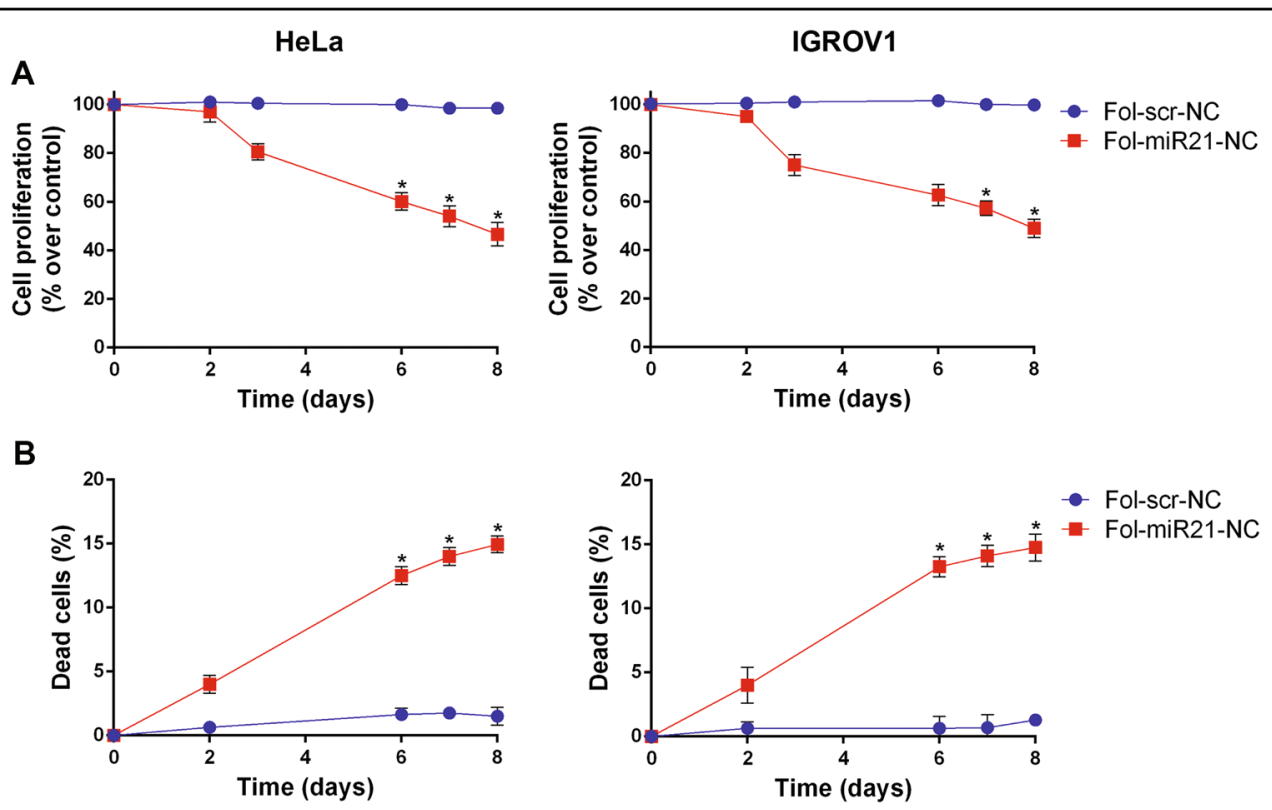

Fig. 6 Cytotoxic effect of Fol-miR21-NCs in HeLa and IGROV1 cells. Cell proliferation after treatment with Fol-NCs at different times was assessed by MTS (A) and cell death was evaluated by trypan blue assay $(\mathbf{B})$. The values are the mean of six replicates normalized to untreated cells. Statistical significance: ${ }^{*} P<0.05$ (Student's $t$-test).
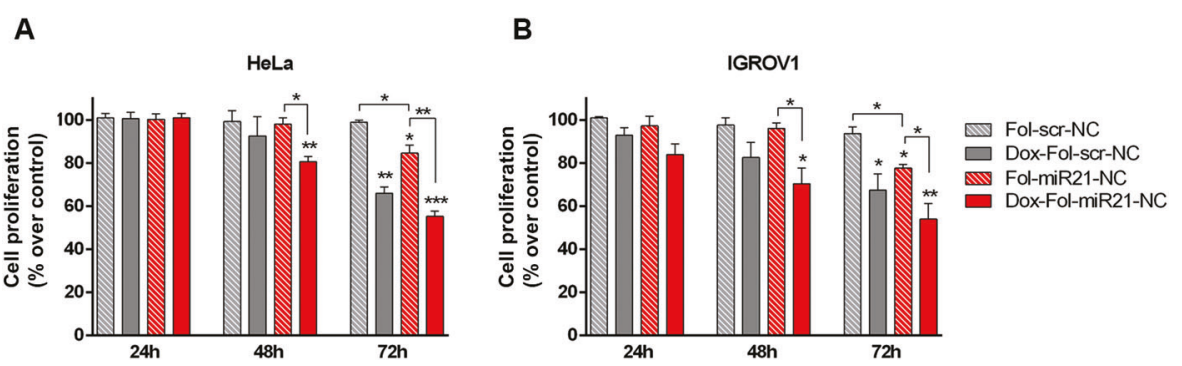

Fig. 7 Effect of Fol-miR21-NCs and Dox combined treatment. Cell proliferation of HeLa (A) and IGROV1 (B) evaluated by MTS assay. Cells were treated with Dox-loaded and not-loaded Fol-miR21-NCs and Fol-scr-NCs at different times, as indicated. The data represent mean \pm S.E.M. of three separate experiments. The values are the mean of six replicates, normalized on cell proliferation of untreated cells. Statistical significance: ${ }^{*} P<0.05{ }^{* * *} P$ $<0.01$, and ${ }^{* * *} P<0.01$ (Student's $t$-test).

induce any toxic effect (see Fig. 6). Therefore, the observed toxicity of control-NCs loaded with doxorubicin (Dox-Fol-scr-NCs), corresponding to $35 \pm 6 \%$ and $32 \pm 9 \%$ of cell viability reduction in HeLa and IGROV1 cells, respectively, is due to the presence of Dox.

\section{Discussion}

Here we demonstrate that Fol-NCs engineered with four sequestering units complementary to miR-21, for inhibiting miR-21 function, enter the cells through the $\alpha F R$-mediated route and sequester miR-21 within cells, leading to $70-80 \%$ reduction in either IGROV1 or HeLa cells, after $48 \mathrm{~h}$ treatment. Considering that the amount of miR-21 present in HeLa cells is $4 \times 10^{3}$ copies $/ 10 \mathrm{pg}$ total
$\mathrm{RNA}^{35}$ corresponding to $0.12 \mathrm{ng} / 10^{6}$ cells and that the amount of internalized cages in HeLa is $200 \mathrm{ng} / 10^{6}$ cells (Fig. 2B), the molar ratio between Fol-miR21-NCs and endogenous miR-21 ranges between 60:1 and 30:1, depending on the experiment. Since each nanocage has four sequestering units, the "miR-21 sequestering units: endogenous miR-21" ratio ranges between 240:1 and $120: 1$, so there are always more than $100 x$ molar excess of sequestering units. This ratio permits an efficient miR-21 binding, as recently demonstrated in vitro ${ }^{31}$.

Folic acid is a biocompatible and non-immunogenic compound internalized in the cytoplasm through $\alpha F R$ pathway for cellular utilization ${ }^{36}$. The advantage of using folate ligand compared to other targeting signals is that, 
when DNA nanostructures enter through the $\alpha \mathrm{FR}$ pathway, they are stable for hours and slowly accumulate inside the cells without degradation ${ }^{26,27}$, likely due to the fact that folic acid is internalized for consumption rather than for destruction ${ }^{36}$. In line, Fig. 2 shows that the highest concentration of intact Fol-NCs inside the cells occurs after $24 \mathrm{~h}$ of incubation, unlike what happens to pristine H4-DNA-NCs administered by transfection ${ }^{31}$. In the latter case, most of the nanocages enter in $2-3 \mathrm{~h}$ and are then quickly degraded.

The Fol-miR21-NC's anti-miR activity, measured by qPCR, is more evident after $48 \mathrm{~h}$ of treatment $(>80 \%$ of inhibition) and miR-21 level remains very low for days, throughout the administration period of the nanostructures. Notably, the inhibition obtained with Fol-NCs is more efficient than that obtained with the anti-miR-21 oligonucleotide given by using a transfection reagent ( $>80 \%$ vs $66 \%$ reduction of miR-21 level over the control) and the anti-miR-21 oligonucleotide transfection has a faster but transient activity. Moreover, transfection agents are toxic ${ }^{37}$ and cannot be administered to cells continuously, whereas folate-functionalized nanocages are not cytotoxic per $\mathrm{se}^{26}$ and can be administered, added to the culture medium, daily for several days (Fig. 6).

Upon confirmation of efficient targeting, we studied the biological effects due to miR-21 silencing. MiR-21 is an overexpressed oncogenic gene in ovarian and cervix cancer and is associated with metastasis and poor prognosis $^{32,33}$. In vitro studies have shown that downregulation of miR-21 results in up-regulation of the tumor suppressor proteins PTEN and Pdcd4, and increased apoptosis ${ }^{32,38}$. In line with these findings, miR21 knockdown by Fol-miR21-NCs leads to a marked upregulation of PTEN and Pdcd4 proteins, respectively.

MiR-21 knockdown by Fol-miR21-NCs leads to inhibition of cell migration evaluated by measuring the scratched area's closure, and to a reduction of cell viability in treated cells. It is interesting to note that both cell lines respond in a similar way to the cytotoxic action. The cell proliferation assays indicate a reduction in cell viability of $51 \%$ and $53 \%$ in HeLa and IGROV1, respectively. In line, similar effects have been obtained using other DNS as vehicles for the release of anti-miR-21 sequences or for direct oncomiRs-silencing activity ${ }^{39-41}$.

Our previous characterization of the intracellular localization of Fol-NCs by confocal analysis ${ }^{26}$ and of their intracellular traffic by colocalization with early endosomes and lysosome markers ${ }^{27}$, indicated that they enter through the folate receptor-mediated endocytosis pathway, allowing their accumulation into the cytoplasm, never going into the nucleus and so excluding that the structures interfere with pri- and pre-miR21 levels.

Finally, Fol-miR21-NCs are an efficient binding platform for intercalating drugs, such as Dox. The loading procedure is easy and the stability of Dox-loaded NCs at physiological $\mathrm{pH}$ indicates to the potential application of DNA nanocages as drug nanocarriers ${ }^{26}$. Folate receptormediated entry of Dox-loaded control nanocages (Fol-scr$\mathrm{NCs})$ results in elevated cytotoxicity, confirming our previous data ${ }^{26}$. Here we show that combination of miR21 sequestering and Dox delivery enhances the cytotoxicity of Fol-miR21-NCs, in an additive way.

In conclusion, the here described self-assembled FolmiR21-NCs, used as delivery vehicle for sequestering intracellular oncogenic miR-21 are very stable and present several advantages. The four oligonucleotides containing miRNA sequestering units can be modified with DNA sequences complementary to different oncomiRs to enlarge the possible applications, and DNA-NCs can be functionalized with different ligands for a selective nontoxic targeting, paving the way for their use as selective nucleic acid drugs. Resistance of cancer cells to therapy along with the occurrence of severe side effects of commonly used treatments have raised the urgency in the development of new and safe anti-cancer agents. Currently, treatments that combine chemotherapeutic drug delivery with gene silencing are emerging as a promising therapeutic option. Multifunctional DNA-based nanocages, here described, engineered to simultaneously achieve selective cell targeting, specific oncomiRs sequestering and drug delivery, can be one of the eligible systems to be applied in cancer therapy to overcome major challenges.

\section{Materials and methods}

\section{Preparation of functionalized octahedral DNA nanocages}

Folate-functionalized nanocages harboring miR-21 sequestering units (Fol-miR21-NCs) or scramble sequences (Fol-scr-NCs) were assembled and purified as described earlier ${ }^{26,31}$. A biotin molecule was added on one edge of the structure for the detection of NCs through the streptavidin (HRP)-biotin reaction. Oligonucleotide sequences used for the assembly of NCs are reported in Supplementary Information 10 and Supplementary Table 1.

\section{Cell cultures}

HeLa cells (provided by M. Figini, Istituto Nazionale Tumori, Milano, Italy) derived from human cervix cancer, were grown in DMEM (Dulbecco's modified Eagle's medium) (Biowest, Miami, FL, USA) and IGROV1 cells (provided by M. Figini, Istituto Nazionale Tumori, Milano, Italy) derived from ovarian carcinoma in RPMI 1640 (Euroclone, Devon, UK), supplemented with 10\% FBS (Gibco, Paisleg, UK), $1 \mathrm{mM}$ L-glutamine (Sigma Aldrich, St Louis, MO, USA), $1 \mathrm{mM}$ sodium pyruvate (Biowest, Miami, FL, USA), and $100 \mathrm{U} / \mathrm{ml}$ penicillin-streptomycin (Euroclone, Devon, UK). Cells were authenticated by STR profiling and periodically tested for mycoplasma contamination. 


\section{Purification of DNA nanocages from cell lysates and DNA blot}

Cells were plated in 48-well plates at a density of $3 \times 10^{4}$ cells/well and grown in folate-free RPMI 1640 (Sigma Aldrich, St Louis, MO, USA) supplemented with 10\% FBS for $24 \mathrm{~h}$. Experiments were performed in folate-free RPMI 1640 medium with 2\% FBS. Cells were lysed, centrifuged, digested with proteinase $\mathrm{K}$, and analyzed by DNA blot, as described earlier ${ }^{25}$. Detection of biotinylated-NCs was carried out using streptavidin-HRP (Horseradish Peroxidase) (Abcam Inc., Toronto, ON, Canada, Ab7403) and visualized by enhanced chemiluminescence (ECL Extend, Euroclone, Devon, UK). Input samples of NCs are DNA structures added to cell culture medium and immediately digested with proteinase $\mathrm{K}$ and processed for DNA blot.

\section{RNA isolation and qPCR for miR-21 expression analysis}

Total RNA was extracted using RNeasy Mini Kit (Qiagen, Hilden, Germany) and reverse transcribed into cDNA by using miScript II RT Kit (Qiagen, Hilden, Germany). RNA concentration was determined using NanoDrop spectrophotometer (NanoDrop ND-1000 Waltham, Massachusetts, MA, USA). For quantitative analysis of miR-21 expression, qPCR amplification of cDNA was performed using miScript Primer assay and miScript SYBR Green PCR Kit (Qiagen, Hilden, Germany) on a Real-Time PCR Detection System (Bio-Rad, Hercules, California,CA, USA), following the manufacturer's instruction. Relative transcript quantification of miR-21 was determined using the $\Delta \Delta \mathrm{Ct}$ method, normalized to the levels of endogenous U6 snRNA and to the untreated control cells.

\section{Western blot}

Cells were lysed and centrifuged as described previously $^{31}$. The supernatant fraction was analyzed by SDSpolyacrylamide gel electrophoresis in 10\% acrylamide gels and blotted (Trans Blot Turbo Bio-Rad Laboratories). PTEN rabbit mAb (Cell Signaling Technologies, Danvers, MA, USA, cat. n. 138G6), Pdcd-4 mouse mAb (Santa Cruz Biotechnology, Dallas, Texas, USA, cat. n. SC376430), and $\beta$-actin mouse mAb (Cell Signaling Technologies, Danvers, MA, USA, cat. n. 8H10D10) were used as primary antibodies. HRP-conjugated AffiniPure goat anti-mouse IgG (Jackson Immunoresearch, Cambridgeshire, UK, cat. n. 115-035-062) and HRP-conjugated AffiniPure donkey anti-rabbit IgG secondary antibody (Jackson Immunoresearch, Cambridgeshire, UK, cat. n. 711-035-152) were used as secondary antibodies. Immunoreactive bands were visualized by enhanced chemiluminescence (ECL Extend, Euroclone, Devon, UK). The ECL data were scanned and analyzed by ImageJ software. Band intensities were normalized to $\beta$-actin.

\section{Doxorubicin intercalation}

Fol-NCs' intercalation with Dox (Enzo Life Sciences Farmingdale, NY, USA) and purification procedure were performed as described previously ${ }^{26}$. The amount of intercalated Dox was calculated as described in Supplementary Information 9.

\section{Cell viability assays}

HeLa and IGROV1 cells were plated in 96-well plates at a density of $2 \times 10^{3}$ cells/well, incubated with folate-free RPMI 1640 with $10 \%$ FBS for $24 \mathrm{~h}$ and treated with FolNCs. Treatment was repeated every $24 \mathrm{~h}$. For longer time points, cells were treated as described above and, after 3 days, detached from wells by trypsinization and plated on 96-well plates. Cells were further treated with Fol-NCs for up to 8 days.

Cell proliferation was evaluated by using the $3-(4,5-$ dimethylthiazol-2-yl)-5-(3-carboxymethoxyphenyl)-2-(4sulfophenyl)-2H-tetrazolium (MTS) assay (Promega, WI, USA). Absorbance was measured at $492 \mathrm{~nm}$ using Multiskan Ascent 96/384 Plate Reader (MTX Lab Systems, Bradenton, FL, USA). Trypan blue staining assay (Sigma Aldrich, St Louis, MO, USA) was performed by using a cell counting chamber.

\section{In vitro migration assay}

HeLa cells were seeded at a density of $1 \times 10^{4}$ cells/well in ibidi Culture-Insert 2 Well (ibidi GmbH, Gräfelfing, Germany) and grown in folate-free RPMI 1640 with 10\% FBS overnight. Cells were incubated with Fol-NCs in folate-free RPMI 1640 with 2\% FBS for $48 \mathrm{~h}$. After the wound was created, the scratch width was measured by photo recordings at 0,12 , and $24 \mathrm{~h}$ using IncuCyte ${ }^{\circledR} \mathrm{S} 3$ Live-Cell Analysis System (Sartorius, Gottinga, Germany) and images captured using a $4 \mathrm{x}$ magnification objective and processed using ImageJ software. Images were converted to grayscale (Image-Type-8bit) and edges enhanced to highlight sharp changes in intensity (Process-Find Edges).

\section{Statistical analysis}

Each experimental point was carried out in duplicate and repeated at least in three independent experiments $(n \geq 6)$. Data were analyzed using GraphPad Prism. Results are expressed as a mean \pm S.E.M. and statistical analyses performed using Student's $t$-test. Differences were considered statistically significant when ${ }^{*} P<0.05$, ** $P<0.01$, and ${ }^{* * *} P<0.001$.

\section{Acknowledgements}

We would like to acknowledge Mariangela Figini (Istituto Nazionale Tumori, Milan, Italy) for providing IGROV1 cell line and for helpful suggestions and Stefano Croce (Baseclick, Neuried, Germany) for providing folate-functionalized OL8 $8_{\mathrm{FOL}}$. 


\section{Funding}

This work was supported by PRIN 2017 Progetto di Rilevante Interesse Nazionale (PRIN) grant (to S.B.), Progetto Ateneo FunDNA and Neuromicro (to A.D.) and Associazione Italiana per la Ricerca sul Cancro, FIRC-AIRC fellowship for Italy (to S.R. and F.I.).

\section{Author contributions}

S.B., A.D., and S.R. designed the project and wrote the paper; S.R., V.U., G.V., C.S. F.I., and S.B. carried out the experimental works and analyzed the data. All authors read and approved the final manuscript.

\section{Conflict of interest}

The authors declare that they have no conflict of interest.

\section{Ethics approval and consent to participate}

The study does not include human participants.

\section{Publisher's note}

Springer Nature remains neutral with regard to jurisdictional claims in published maps and institutional affiliations.

Supplementary Information accompanies this paper at (https://doi.org/ 10.1038/s41419-020-03339-3).

Received: 20 July 2020 Revised: 8 December 2020 Accepted: 10 December 2020

Published online: 07 January 2021

\section{References}

1. Bartel, D. P. MicroRNAs: genomics, biogenesis, mechanism, and function. Cell 116, 281-297 (2004)

2. Iorio, M. V. et al. MicroRNA signatures in human ovarian cancer. Cancer Res. 67 8699-8707 (2007)

3. Yang, H. et al. MicroRNA expression signatures in Barrett's esophagus and esophageal adenocarcinoma. Clin. Cancer Res. 15, 5744-5752 (2009).

4. Kim, Y. W. et al. Differential microRNA expression signatures and cell typespecific association with Taxol resistance in ovarian cancer cells. Drug Des. Devel. Ther. 8, 293-314 (2014).

5. Calin, G. A. et al. MicroRNA profiling reveals distinct signatures in B cell chronic lymphocytic leukemias. Proc. Natl Acad. Sci. USA 101, 11755-11760 (2004).

6. Qi, J., Wang, J., Katayama, H., Sen, S. \& Liu, S. M. Circulating microRNAs (cmiRNAs) as novel potential biomarkers for hepatocellular carcinoma. Neoplasma 60, 135-142 (2013).

7. lorio, M. V. et al. MicroRNA gene expression deregulation in human breast cancer. Cancer Res. 65, 7065-7070 (2005).

8. Volinia, S. et al. A microRNA expression signature of human solid tumors defines cancer gene targets. Proc. Natl Acad. Sci. USA 103, 2257-2261 (2006).

9. Bautista-Sánchez, D. et al. The promising role of miR-21 as a cancer biomarker and its importance in RNA-based therapeutics. Mol. Ther. Nucleic Acids $\mathbf{2 0}$ 409-420 (2020).

10. Krichevsky, A. M. \& Gabriely, G. miR-21: a small multi-faceted RNA. J. Cell. Mol. Med. 13, 39-53 (2009).

11. Lee, T. J. et al. RNA nanoparticle-based targeted therapy for glioblastoma through inhibition of oncogenic miR-21. Mol. Ther. 25, 1544-1555 (2017).

12. Griveau, A. et al. Silencing of miR-21 by locked nucleic acid-lipid nanocapsule complexes sensitize human glioblastoma cells to radiation-induced cell death. Int. J. Pharm. 454, 765-774 (2013).

13. $\mathrm{Hu}, \mathrm{Q} ., \mathrm{L}, \mathrm{H} .$, Wang, L., Gu, H. \& Fan, C. DNA nanotechnology-enabled drug delivery systems. Chem. Rev. 119, 6459-6506 (2019).

14. Zhu, B., Wang, L., Li, J. \& Fan, C. Precisely tailored DNA nanostructures and their theranostic applications. Chem. Rec. 17, 1213-1230 (2017).

15. Angell, C., Xie, S., Zhang, L. \& Chen, Y. DNA nanotechnology for precise control over drug delivery and gene therapy. Small 12, 1117-1132 (2016).
16. Franch, O. et al. DNA hairpins promote temperature controlled cargo encapsulation in a truncated octahedral nanocage structure family. Nanoscale 8, 13333-13341 (2016)

17. Schaffert, D. H. et al. Intracellular delivery of a planar DNA origami structure by the transferrin-receptor internalization pathway. Small 12, 2634-2640 (2016)

18. Raniolo, S. et al. Cellular uptake of covalent and non-covalent DNA nanostructures with different sizes and geometries. Nanoscale 11, 10808-10818 (2019).

19. Linko, V., Ora, A. \& Kostiainen, M. A. DNA nanostructures as smart drug-delivery vehicles and molecular devices. Trends Biotechnol. 33, 586-594 (2015).

20. Xie, N. et al. DNA tetrahedron nanostructures for biological applications: biosensors and drug delivery. Analyst 142, 3322-3332 (2017).

21. Lee, H. et al. Molecularly self-assembled nucleic acid nanoparticles for targeted in vivo siRNA delivery. Nat. Nanotechnol. 7. 389-393 (2012).

22. Andersen, F. F. et al. Assembly and structural analysis of a covalently closed nano-scale DNA cage. Nucleic Acids Res. 36, 1113-1119 (2008).

23. Falconi, $\mathrm{M}$. et al. Deciphering the structural properties that confer stability to a DNA nanocage. ACS Nano 3, 1813-1822 (2009).

24. Oliveira, C. L. P. et al. Structure of nanoscale truncated octahedral DNA cages: variation of single-stranded linker regions and influence on assembly yields. ACS Nano 4, 1367-1376 (2010)

25. Vindigni, $\mathrm{G}$. et al. Receptor-mediated entry of pristine octahedral DNA nanocages in mammalian cells. ACS Nano 10, 5971-5979 (2016).

26. Raniolo, S. et al. Selective targeting and degradation of doxorubicin-loaded folate-functionalized DNA nanocages. Nanomedicine 14, 1181-1190 (2018).

27. Raniolo, S. et al. Entry, fate and degradation of DNA nanocages in mammalian cells: a matter of receptors. Nanoscale 10, 12078-12086 (2018).

28. Cheung, A. et al. Targeting folate receptor alpha for cancer treatment. Oncotarget 7, 52553-52574 (2016).

29. Tacar, O., Sriamornsak, P. \& Dass, C. R. Doxorubicin: an update on anticancer molecular action, toxicity and novel drug delivery systems. J. Pharm. Pharmacol. 65, 157-170 (2013).

30. Yang, F., Teves, S. S., Kemp, C. J., Henikoff, S. Doxorubicin, DNA torsion, and chromatin dynamics. Biochim. Biophys. Acta 1845, 84-89 (2014).

31. Raniolo, S., lacovelli, F., Unida, V., Desideri, A. \& Biocca, S. In Silico and in cell analysis of openable DNA nanocages for miRNA silencing. Int. J. Mol. Sci. 21, 61 (2019).

32. $\mathrm{Xu}$, J., Zhang, W., LV, Q. \& Zhu, D. Overexpression of miR-21 promotes the proliferation and migration of cervical cancer cells via the inhibition of PTEN. Oncol. Rep. 33, 3108-3116 (2015).

33. Zhang, L., Nadeem, L., Connor, K. \& Xu, G. Mechanisms and therapeutic targets of microRNA-associated chemoresistance in epithelial ovarian cancer. Curr. Cancer Drug Targets 16, 429-441 (2016).

34. Bagnoli, M. et al. Downmodulation of caveolin-1 expression in human ovarian carcinoma is directly related to alpha-folate receptor overexpression. Oncogene 19, 4754-4763 (2000)

35. Androsavich, J. R., Chau, B. N., Bhat, B., Linsley, P. S. \& Walter, N. G. Diseaselinked microRNA-21 exhibits drastically reduced mRNA binding and silencing activity in healthy mouse liver. RNA 18, 1510-1526 (2012).

36. Wang, S., Lee, R. J., Cauchon, G., Gorenstein, D. G. \& Low, P. S. Delivery of antisense oligodeoxyribonucleotides against the human epidermal growth factor receptor into cultured KB cells with liposomes conjugated to folate via polyethylene glycol. Proc. Natl Acad. Sci. USA 92, 3318-3322 (1995).

37. Wang, T. Larcher, L. M., Ma, L. \& Veedu, R. N. Systematic screening of commonly used commercial transfection reagents towards efficient transfection of single-stranded oligonucleotides. Molecules 23, 2564 (2018).

38. Yao, Q., Xu, H., Zhang, Q. Q., Zhou, H. \& Qu, L. H. MicroRNA-21 promotes cell proliferation and down-regulates the expression of programmed cell death 4 (PDCD4) in HeLa cervical carcinoma cells. Biochem. Biophys. Res. Commun. 388, 539-542 (2009)

39. Xue, H. et al. DNA tetrahedron-based nanogels for siRNA delivery and gene silencing. Chem. Commun. (Camb) 55, 4222-4225 (2019).

40. Liu, Q. et al. Capturing intracellular oncogenic microRNAs with self-assembled DNA nanostructures for microRNA-based cancer therapy. Chem. Sci. 9, 7562-7568 (2018)

41. Su, J., Wu, F., Xia, H., Wu, Y. \& Liu, S. Accurate cancer cell identification and microRNA silencing induced therapy using tailored DNA tetrahedron nanostructures. Chem. Sci. 11, 80-86 (2019). 